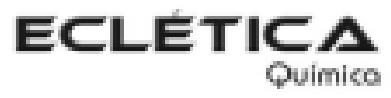

www.scielo.br/eq

Volume 29, número 1, 2004

\title{
Determinação de parâmetros físico-químicos com um sensor potenciométrico sensível ao íon p-aminobenzoato
}

\author{
A. O. Santini ${ }^{1}$, E. S. da Silva Júnior ${ }^{1}$, H. R. Pezza ${ }^{1}$ L. Pezza ${ }^{1}$ \\ ${ }^{'}$ Instituto de Química - UNESP - CEP 14800-900 Araraquara - SP - Brasil.
}

Resumo: Este trabalho descreve a construção e a determinação de parâmetros físico-químicos com um eletrodo de segunda ordem do tipo, $\mathrm{Pt}|\mathrm{Hg}| \mathrm{Hg}_{2}(\mathrm{PAB})_{2} \mid$ Grafite, sensível ao íon p-aminobenzoato (PAB). $\mathrm{O}$ eletrodo é construído facilmente, apresenta um rápido tempo de resposta, é de baixo custo e tem um tempo de vida útil superior a 12 meses. Utilizando o referido eletrodo foi possível estimar os coeficientes de atividade iônica individuais do $\mathrm{PAB}$, a $25^{\circ} \mathrm{C}$, em força iônica ajustada entre $0,700-3,000 \mathrm{~mol} \mathrm{~L}^{-1} \mathrm{com}$ $\mathrm{NaClO}_{4}$, em solução aquosa. O potencial molal padrão do eletrodo determinado a $25^{\circ} \mathrm{C}$ é de $(445,5 \pm 0,5)$ $\mathrm{mV}$. A constante termodinâmica do produto de solubilidade, ${ }^{\mathrm{T}} \mathrm{K}_{\mathrm{ps}}(\mathrm{I}=0) \mathrm{do} \mathrm{Hg}_{2}(\mathrm{PAB})_{2}$ determinada com o eletrodo é de $\left({ }^{\mathrm{T}} \mathrm{K}_{\mathrm{ps}}=2,50 \times 10^{-12} \mathrm{~mol}^{3} \mathrm{~L}^{-3}\right.$, a $\left.25^{\circ} \mathrm{C}\right)$. Uma característica favorável consiste no fato de que o eletrodo pode ser utilizado para determinar a concentração de íons p-aminobenzoato livres em sistemas complexos de íons metálicos.

Palavras-chave: sensor potenciométrico; p-aminobenzoato; constante termodinâmica do produto de solubilidade; coeficiente de atividade individual iônico

\section{Introdução}

O ácido p-aminobenzóico (APAB) é um composto antioxidante produzido em nosso organismo que desempenha importante papel na assimilação do ácido pantotênico, na formação de glóbulos vermelhos, sendo nutriente essencial para as bactérias intestinais atuando como precursor na síntese de ácido fólico [13]. Sua ausência pode causar extrema fadiga, eczema, irritabilidade, depressão, nervosismo, constipação, perturbações digestivas, dores de cabeça, etc.

O APAB é muito empregado em preparações vitamínicas e produtos geriátricos como, por exemplo, o gerovital $\mathrm{H}_{3}(\mathrm{GH} 3)$ e usado no tratamento de fibroses decorrentes de certas doenças dermatológicas como a doença de peyronie, escleroderma, além de auxiliar o retorno dos cabelos grisalhos à sua cor natural quando a mudança for causada por stress ou deficiência nutricional.[18-19]
Também é empregado na manufatura de vários ésteres (anestésicos locais), de ácido fólico, de corantes e de medicamentos dermatológicos. Além disso, o APAB e seus derivados pertencem a uma categoria muito popular de substâncias (principalmente nos EUA e na Escandinávia) que são apropriadas para filtrar os nocivos raios, UV-B provenientes da radiação solar [3]. A camada de ozônio está sendo destruída gradualmente pelo homem e dentro desse contexto, um agente de proteção solar deve ser usado para minimizar os efeitos causados pela exposição aos raios UV que são nocivos à pele, além de serem agentes carcinogênicos [5.] Portanto, a adição de um agente de proteção solar (como o APAB) aos protetores solares e aos cosméticos como loções, óleos e bálsamos é essencial.

A extração do $\mathrm{APAB}$ em cosméticos é feita com solventes e a sua determinação pode ser realizada por vários métodos [41]. Tais métodos são baseados principalmente em cromatografia líqui- 
da [24], cromatografia líquida de alta eficiência [20,39] e cromatografia gasosa.[21]

Uma das linhas desse grupo de pesquisa envolve estudos de equilíbrios em solução, cuja finalidade principal é a obtenção de relações matemáticas entre as constantes de formação de sistemas constituídos por íons metálicos e ligantes orgânicos (geralmente carboxilatos) de interesse biológico, farmacológico, ambiental, alimentício [1], etc., em solução aquosa, com a composição e a força iônica (I) do meio.[22,25,30,32]

Dentro desse contexto, torna-se necessário determinar a dependência dos coeficientes de atividade de todas as espécies envolvidas nos equilíbrios com a força iônica do meio [27,30-33]. Uma maneira conveniente e elegante de se atingir tal propósito é através do emprego de eletrodos sensíveis a íons metálicos e de eletrodos sensíveis a ligantes orgânicos.

Os eletrodos sensíveis a ligantes orgânicos (carboxilatos) podem ser empregados em medidas de potencial de uma célula potenciométrica (onde estes, configuram-se como eletrodos indicadores) as quais podem fornecer dados que permitem a determinação do coeficiente de atividade iônica do ligante orgânico correspondente em função da força iônica do meio.

A constante de equilíbrio para uma reação que possa ser potencialmente empregada numa titulação de precipitação pode ser, aproximadamente, deduzida do valor da constante do produto de solubilidade $\left(\mathrm{K}_{\mathrm{ps}}\right)$. $\mathrm{O} \mathrm{K}_{\mathrm{ps}}$ relaciona-se especificamente ao equilíbrio que é estabelecido entre um sólido iônico escassamente solúvel e seus íons numa solução saturada do sólido.

Os produtos de solubilidade são, geralmente, determinados por medidas de solubilidade do sólido iônico, no meio de interesse. Tal procedimento não é muito conveniente, uma vez que envolve etapas de filtração e centrifugação. [35]

Um outro método comumente usado na determinação dos valores de $\mathrm{K}_{\mathrm{ps}}$ envolve o emprego de titulações potenciométricas de precipitação, o qual, embora mais preciso que o anteriormente descrito, também apresenta limitações [23,26].

O presente trabalho mostra o desenvolvimento e aplicação físico-química de um sensor potenciométrico sensível ao íon p-aminobenzoato imobilizado em matriz de grafite (na forma de pas- tilha prensada) do tipo $\mathrm{Pt}|\mathrm{Hg}| \mathrm{Hg}_{2}(\mathrm{PAB})_{2} \mid$ Grafite. Medidas potenciométricas com o referido eletrodo em soluções de íons p-aminobenzoato, em função da variação da força iônica do meio $(0,700 £ \mathrm{I} £$ $3,000 \mathrm{~mol} \mathrm{~L}^{-1} \mathrm{em} \mathrm{NaClO}_{4}, \mathrm{a} 25^{\circ} \mathrm{C}$ ), possibilitaram a determinação do potencial padrão do eletrodo indicador, da constante termodinâmica do produto de solubilidade $\left({ }^{\mathrm{T}} \mathrm{K}_{\mathrm{ps}}\right.$ ) do p-aminobenzoato mercuroso, bem como uma estimativa dos coeficientes de atividade iônica individuais do íon paminobenzoato.

\section{Procedimento Experimental}

Todos os reagentes utilizados na execução do presente trabalho foram de pureza analítica (p.a.). Na preparação e padronização das soluções foi utilizada água destilada e desionizada no sistema NANOpure II (Sybron Barnstead) além de vidraria grau "A". O mercúrio usado foi purificado e destilado em nosso laboratório segundo procedimento anteriormente descrito [27]. Todas as operações foram realizadas em sala climatizada a $(25,0$ $\pm 1,0){ }^{\circ} \mathrm{C}$, onde encontram-se instalados os equipamentos de medida. Todo o resíduo ou solução descartável contendo mercúrio (em qualquer estado de oxidação) foram mantidos em reservatórios apropriados para descarte de metais pesados.

\section{Síntese do p-aminobenzoato mercuroso}

$\mathrm{O}$ p-aminobenzoato mercuroso foi sintetizado adicionando-se lentamente e, sob agitação, $50,00 \mathrm{~mL}$ de uma solução de $\mathrm{Hg}_{2}\left(\mathrm{NO}_{3}\right)_{2} 0,644$ $\mathrm{molL}^{-1}$ a $100,00 \mathrm{~mL}$ de uma solução de paminobenzoato de sódio $0,500 \mathrm{molL}^{-1}$. Deixou-se sob agitação por mais alguns minutos para completa precipitação do sal. O precipitado formado foi filtrado em funil de vidro sinterizado e lavado com água deionizada para eliminar o excesso de p-aminobenzoato de sódio e deixado até completa secagem em dessecador (contendo $\mathrm{CaCl}_{2}$ como secante) à pressão reduzida.

O sal mercuroso obtido, $\mathrm{Hg}_{2}(\mathrm{PAB})_{2}$, apresentou-se na forma de um fino pó branco e foi utilizado na construção do eletrodo de $\mathrm{Pt}|\mathrm{Hg}| \mathrm{Hg}_{2}(\mathrm{PAB})_{2} \mid$ Grafite. 


\section{Construção do eletrodo $\mathrm{Pt}|\mathrm{Hg}| \mathrm{Hg}_{2}(\mathrm{PAB})_{2} \mid$ Grafite}

A imobilização da pasta sensora $\mathrm{Hg} \mid \mathrm{Hg}_{2}(\mathrm{PAB})_{2}$ em matriz de grafite foi efetuada, partindo-se de aproximadamente $1,30 \mathrm{~g}$ de $\mathrm{p}$ aminobenzoato mercuroso, $0,65 \mathrm{~g}$ de grafite em pó e 1 gota de mercúrio metálico, sendo os respectivos reagentes misturados e triturados em almofariz de ágata até a obtenção de uma pasta homogênea. Cerca de $0,70 \mathrm{~g}$ do pó preparado foi transferido para um molde de aço de $10 \mathrm{~mm}$ de diâmetro interno e espessura de $1,5 \mathrm{~mm}$, prensado em um pastilhador Marconi, a 6,5 toneladasforça por 10 minutos. Testes previamente realizados mostraram que a composição 2:1 (paminobenzoato-grafite), bem como as condições de pastilhamento acima citadas foram as mais adequadas para a construção do eletrodo de paminobenzoato.

A pastilha obtida (de cor preta) pesando cerca de 0,60 g, com um diâmetro interno de 10 $\mathrm{mm}$ e espessura de $1,5 \mathrm{~mm}$ foi fixada na extremidade de um tubo de vidro com cola de borracha de silicone ("Rhodiastic", Rhodia) e deixada secar por 48 horas. $\mathrm{O}$ contato elétrico foi estabelecido através de um fio de platina imerso dentro do poço de $\mathrm{Hg}^{\mathrm{o}}$ e um subseqüente cabo condutor com um plug do tipo "banana" ou mini-din tipo F conectado a dispositivo de teflon adaptável ao eletrodo e que permite a sua vedação. Pelas características do eletrodo (que é vedado) e pela utilização esporádica de pequenas quantidades de $\mathrm{Hg}^{\circ}$ (sempre manipulado em capela de boa exaustão), pode-se afirmar que a exposição do operador ao $\mathrm{Hg}^{\mathrm{O}}$ é negligenciável. O eletrodo resultante está esquematizado na Figura 1.

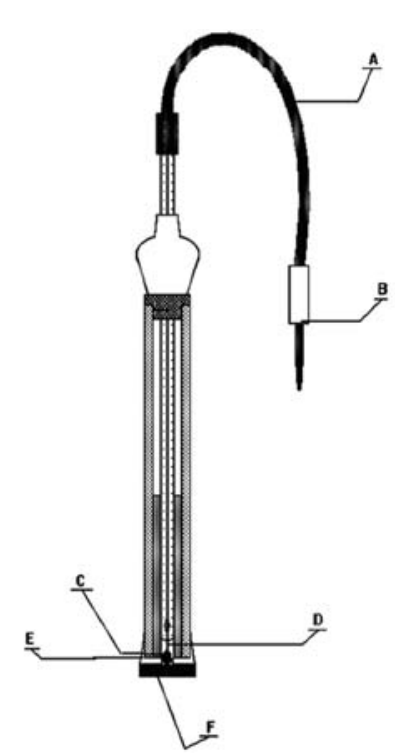

Figura 1 - Eletrodo $\mathrm{Pt}|\mathrm{Hg}| \mathrm{Hg}_{2}(\mathrm{PAB})_{2} \mid$ Grafite, PAB: paminobenzoato;

A: cabo condutor; B: plug banana; C: mercúrio metálico; D: fio de platina; E: cola de silicone; F: patilha sensora (Grafite $\left|\mathrm{Hg}_{2}(\mathrm{PAB})_{2}\right| \mathrm{Hg}$ ).

Método utilizado para aquisição dos dados experimentais

As medidas potenciométricas foram realizadas mediante o uso de um pHmetro "Metrohm" 692 (com precisão de $\pm 0,1 \mathrm{mV}$ ), acoplado a buretas de pistão automáticas "Metrohm", $\bmod 665$ (com precisão de $\pm 0,001 \mathrm{~mL}$ ), utilizando-se uma célula potenciométrica de paredes duplas de vidro ("Metrohm", n' 6.1418.220) termostatizada a (25,0 $\pm 0,1)^{\circ} \mathrm{C}$. A seguinte célula potenciométrica foi utilizada:

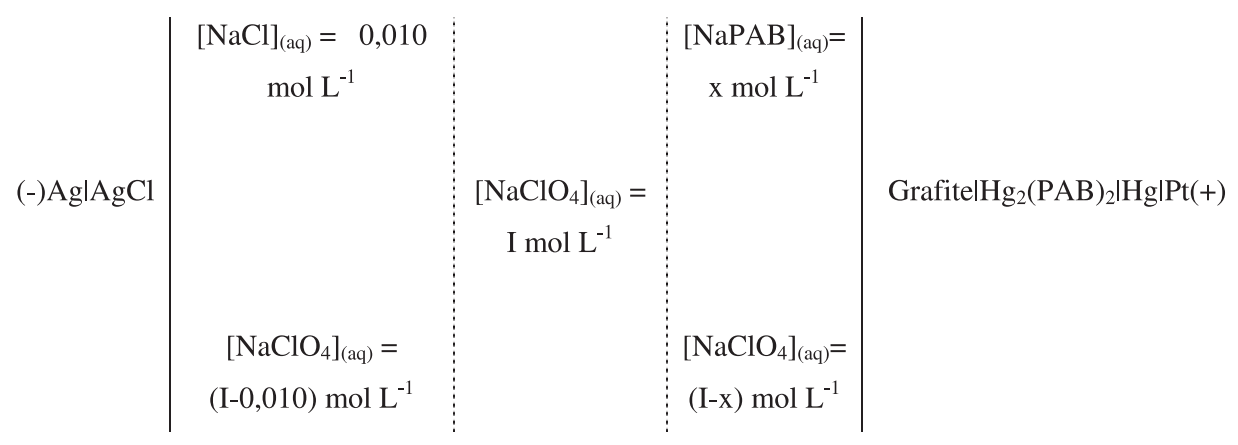


onde: $x$ corresponde a uma determinada concentração de NaPAB (p-aminobenzoato). A força iônica da solução foi mantida constante num determinado valor pela adição de $\mathrm{NaClO}_{4}$.

Os valores experimentais de força eletromotriz, $\mathrm{E}_{\text {cel }}$, numa dada força iônica $\left(\mathrm{I}_{\text {total }}=\mathrm{X}\right.$ molL ${ }^{-1}, 0,700 \mathrm{~mol} \mathrm{~L}^{-1} £ \mathrm{X} £ 3,000 \mathrm{molL}^{-1}$ ajustado com $\mathrm{NaClO}_{4}$ ) foram obtidos pela adição sucessiva de incrementos constantes de volumes $(0,200 \mathrm{~mL}$ até um total de $5,000 \mathrm{~mL}$ ) de uma solução 0,100 mol L-1 de p-aminobenzoato de sódio $(\mathrm{pH}=7,0$, $\mathrm{I}_{\text {total }}=\mathrm{X} \mathrm{mol} \mathrm{L} \mathrm{L}^{-1}$ em $\mathrm{NaClO}_{4}$ ) à uma célula potenciométrica com paredes duplas de vidro contendo $15,000 \mathrm{~mL}$ de perclorato de sódio $(\mathrm{pH}=7,0)$ e $0,100 \mathrm{~mL}$ de p-aminobenzoato de sódio 0,100 $\mathrm{mol} \mathrm{L}^{-1}(\mathrm{pH}=7,0)$ ambos com força iônica $\mathrm{X}$ mol $\mathrm{L}^{-1}$. Após cada adição $(\mathrm{t}=30 \mathrm{~s})$ sob agitação contínua, a força eletromotriz da célula descrita foi medida.

Uma pequena quantidade de paminobenzoato de sódio foi inicialmente adicionada $\left(0,100 \mathrm{~mL} ; 0,100 \mathrm{molL}^{-1}\right)$ para promover uma melhor estabilidade de leitura.

$\mathrm{O}$ eletrodo de referência utilizado foi $\mathrm{Ag} /$ $\mathrm{AgCl}, \mathrm{NaCl}\left(0,010 \mathrm{~mol} \mathrm{~L}^{-1}\right)$ de procedência "Metrohm", no 6.0726.100 de dupla junção (associado a uma ponte salina vertical).

\section{Tratamento dos dados experimentais}

A força eletromotriz $\left(\mathrm{E}_{\text {cel. }}\right)$ da célula pode ser dada por: $[6,31,32]$

$\mathrm{E}_{\text {cel. }}=\mathrm{E}_{\mathrm{ind}}-\mathrm{E}_{\mathrm{ref}}+\mathrm{E}_{\mathrm{j}}$

$\mathrm{E}_{\text {ind }}=$ potencial do eletrodo indicador.

$\mathrm{E}_{\mathrm{ref}}=$ potencial do eletrodo de referência.

$\mathrm{E}_{\mathrm{j}}=$ potencial de junção líquida (desprezível nas condições experimentais empregadas).

ou por:

$\mathrm{E}_{\text {cel. }}=\left(\mathrm{E}_{\mathrm{PAB}}{ }^{\circ}\right)_{0}-\mathrm{S} \log \mathrm{a}_{\mathrm{PAB}}$

onde:

$\mathrm{a}_{\mathrm{PAB}}=\mathrm{y}_{\mathrm{PAB}} \cdot[\mathrm{PAB}] ; \mathrm{y}_{\mathrm{PAB}}=$ coeficiente de atividade molar do íon PAB (p-aminobenzoato).

$[\mathrm{PAB}]=$ concentração do íon $\mathrm{PAB}\left(\mathrm{mol} \mathrm{L}^{-1}\right), \mathrm{PAB}$ (p-aminobenzoato).

$\mathrm{S}=$ coeficiente angular da curva analítica (coeficiente de Nernst).

$\left(\mathrm{E}_{\mathrm{PAB}}^{\mathrm{o}^{\prime}}\right)_{\mathrm{o}}=\mathrm{E}_{\mathrm{Hg} 2 \mathrm{PAB} \mid \mathrm{Hg}}^{\mathrm{o}}-\mathrm{E}_{\mathrm{ref}}+\mathrm{E}_{\mathrm{j}}$, sendo:

$\mathrm{E}_{\mathrm{Hg} 2(\mathrm{PAB}) 2 / \mathrm{Hg}}^{\mathrm{o}}=$ potencial padrão do eletrodo indicador.

$\mathrm{E}_{\mathrm{ref}}=$ potencial do eletrodo de referência.

$E_{j}=X_{P A B}$. $[P A B]=$ potencial de junção líquida (desprezível nas condições experimentais empregadas). $[6,32]$

$\mathrm{X}_{\mathrm{PAB}}=$ coeficiente do potencial de junção líquida.

A equação (1a) pode ser desmembrada:

$\mathrm{E}_{\text {cel. }}=\left(\mathrm{E}_{\mathrm{PAB}}{ }^{\mathrm{o}^{\prime}}\right)_{\mathrm{o}}-\mathrm{S} \log \mathrm{y}_{\mathrm{PAB}}-\mathrm{S} \log [\mathrm{PAB}]$

Considerando-se o potencial condicional da célula como:

$\mathrm{E}_{\mathrm{PAB}}^{\mathrm{o}^{\prime}}=\left(\mathrm{E}_{\mathrm{PAB}}^{\mathrm{o}^{\prime}}\right)_{0}-\mathrm{S} \log \mathrm{y}_{\mathrm{PAB}}$

Supondo $\mathrm{y}_{\mathrm{PAB}}=$ constante (quando $\mathrm{I}=$ constante e o eletrólito de suporte está presente em concentração suficientemente majoritária), tem-se:

$\mathrm{E}_{\text {cel. }}=\mathrm{E}_{\mathrm{PAB}}{ }^{\mathrm{o}^{\prime}}-\mathrm{S} \log [\mathrm{PAB}]$

A partir de dados experimentais (-log [PAB], $E_{\text {cel. }}$ ) os parâmetros $E_{P A B}{ }^{0^{\prime}}$ e $S$ foram determinados em cada força iônica estudada, mediante método de regressão linear.

A expressão genérica utilizada para obter os valores de $\log \mathrm{y}_{\mathrm{PAB}}$, válida para misturas de eletrólitos, [14, 30 ,32]é a seguinte :

$\mathrm{f}_{\mathrm{PAB}}=\log \mathrm{y}_{\mathrm{PAB}}=\mathrm{a} \mathrm{I}^{1 / 2}+\mathrm{bI}+\mathrm{cl} \mathrm{l}^{3 / 2}+\mathrm{dI}^{2}+\ldots=\mathrm{f}_{\mathrm{PAB}}$

onde: a,b,c,d... são parâmetros empíricos ou semiempíricos.

Combinando-se as equações (3) e (5), têm-se:

$\mathrm{E}_{\mathrm{PAB}}{ }^{\mathrm{o}^{\prime}}=\left(\mathrm{E}_{\mathrm{PAB}}{ }^{\mathrm{o}^{\prime}}\right)_{\mathrm{o}}-\mathrm{S}\left(\mathrm{aI}^{1 / 2}+\mathrm{bI}+\mathrm{cl}^{3 / 2}+\mathrm{dI}^{2}+\ldots.\right)$ 
Dispondo-se de suficientes pares de valores $\left(E_{P A B}{ }^{\circ}, I\right)$, foram determinados os parâmetros envolvidos na equação (5) e portanto a dependência entre $\mathrm{y}_{\mathrm{PAB}}$ e a força iônica do meio a $25^{\circ} \mathrm{C}$.

Através das curvas analíticas experimentais, foi possível estimar o valor do potencial formal da célula $\left(\mathrm{E}^{0^{\circ}}{ }^{\circ e l}=E^{0^{\prime}}{ }_{\mathrm{PAB}}\right)$ para cada valor de I, empregando-se a equação (4). A representação gráfica dos pares $\left(\mathrm{E}^{\circ}{ }_{\mathrm{PAB}}, \mathrm{I}\right)$ permite o ajuste de uma equação matemática que represente a variação dos $\mathrm{E}^{\mathrm{o}^{\prime}}{ }_{\mathrm{cel}}$ $=\mathrm{E}^{0^{\prime}}{ }_{\mathrm{PAB}}$ com a força iônica do meio, cuja extrapolação para $\mathrm{I}=0$ fornece $\mathrm{o}$ valor $\left(\mathrm{E}^{\mathrm{o}^{\prime}}{ }_{\mathrm{cel}}\right)_{\mathrm{o}}=\left(\mathrm{E}_{\mathrm{PAB}}^{\mathrm{o}^{\prime}}\right)_{\mathrm{o}}$

$\left(\mathrm{E}_{\mathrm{PAB}}^{\circ}\right)_{\mathrm{o}}=\mathrm{E}_{\mathrm{Hg} / 2 \mathrm{Hg} 2(\mathrm{PAB}) 2}^{\circ}-\mathrm{E}_{\mathrm{ref}}$

Quando $\mathrm{I}=0, \mathrm{~g}_{\mathrm{Cl}}{ }^{-}=1$ e $\mathrm{a}_{\mathrm{Cl}}{ }^{-}=\mathrm{C}_{\mathrm{Cl}}$. Assim, nas condições experimentais empregadas $\left(\mathrm{C}_{\mathrm{Cl}}{ }^{-}=\right.$ $\left.0,010 \mathrm{~mol} \mathrm{~L}^{-1}\right)$ o potencial do eletrodo de referência $(\mathrm{Ag} \mid \mathrm{AgCl})$ é: $\mathrm{E}_{\text {ref }}=340,5 \mathrm{mV}$.

Portanto, o valor do potencial padrão do eletrodo indicador ( $\mathrm{p}$-aminobenzoato) pode ser determinado pela equação (7), e conseqüentemente o valor de ${ }^{\mathrm{T}} \mathrm{K}_{\mathrm{ps}}$ (termodinâmico) do p-aminobenzoato mercuroso a partir de $\mathrm{E}_{\mathrm{Hg} / 2 \mathrm{Hg} 2(\mathrm{PAB}) 2}^{\mathrm{o}}$ e do $\mathrm{E}_{\mathrm{Hg}}^{\mathrm{o}}{ }^{2+}{ }_{\mathrm{Hg}}$ (790 mV), utilizando-se a equação[28]:

$\log { }^{\mathrm{T}} \mathrm{K}_{\mathrm{ps}}=2\left[\left(\mathrm{E}_{\mathrm{Hg} / \mathrm{Hg} 2(\mathrm{PAB}) 2}^{\mathrm{o}}-\mathrm{E}_{\mathrm{Hg} 2}^{\mathrm{o}} /{ }_{\mathrm{Hg}}^{2+}\right) / 59,16\right]$

\section{Resultados e discussão}

O tempo de resposta do eletrodo é de 30 segundos, e foi determinado segundo experimento descrito anteriormente[34].

As curvas analíticas foram obtidas para cada força iônica estudada, e após o ajuste pelo método de regressão linear, chegaram-se às equações e aos intervalos de concentração de p-aminobenzoato para cada força iônica. Embora a faixa linear do eletrodo seja mais ampla $\left(5,0 \times 10^{-4} \mathrm{~mol} \mathrm{~L}^{-1}\right.$ a $1,0 \mathrm{x}$ $10^{-1} \mathrm{~mol} \mathrm{~L}^{-1}$ ), empregou-se uma faixa mais estreita de concentração nos experimentos físico-químicos com o objetivo de diminuir o tempo necessário para a construção das curvas analíticas do eletrodo. Os dados obtidos são apresentados na tabela 1 .

Tabela 1 - Parâmetros das equações das curvas analíticas do eletrodo $\mathrm{Pt}^{1} / 2 \mathrm{Hg}^{1} / 2 \mathrm{Hg}_{2}(\mathrm{PAB})_{2}^{1 / 2 \mathrm{Grafite}}$, seus respectivos intervalos de $[\mathrm{PAB}]$ e o coeficiente de correlação da reta, para cada força iônica. Temperatura $=(25 \pm 0,1)^{\circ} \mathrm{C}$.

\begin{tabular}{|c|c|c|c|c|c|c|}
\hline \multirow[t]{2}{*}{$\begin{array}{c}\mathrm{I} \\
\left(\mathrm{mol} \mathrm{L}^{-1}\right)\end{array}$} & \multirow[t]{2}{*}{$\begin{array}{l}\text { Intervalo de [PAB] estudado } \\
\left(\mathrm{mol} \mathrm{L}^{-1}\right)\end{array}$} & \multicolumn{4}{|c|}{$\begin{array}{l}\text { Parâmetros da equação das curvas analíticas } \\
\text { conforme equação (4) }\end{array}$} & \multirow[t]{2}{*}{$r^{(d)}$} \\
\hline & & $E_{P A B}{ }^{\circ{ }^{\prime \prime}(a)}$ & $\mathrm{DP}^{(6)}$ & $\mathrm{S}^{(\mathrm{c})}$ & $\mathrm{DP}^{(\mathrm{b})}$ & \\
\hline 0,700 & $6,83 \times 10^{-3}$ a $2,54 \times 10^{-2}$ & 108,3 & 1,08 & 53,5 & 0,6 & 0,9987 \\
\hline 0,900 & $1,53 \times 10^{-2}$ a $2,54 \times 10^{-2}$ & 109,4 & 0,98 & 58,3 & 0,5 & 0,9991 \\
\hline 1,200 & $1,53 \times 10^{-2}$ a $2,54 \times 10^{-2}$ & 111,1 & 0,96 & 57,9 & 0,5 & 0,9991 \\
\hline 1,500 & $1,53 \times 10^{-2}$ a $2,54 \times 10^{-2}$ & 112,7 & 0,85 & 59,9 & 0,5 & 0,9990 \\
\hline 1,800 & $5,66 \times 10^{-3}$ a $2,54 \times 10^{-2}$ & 113,0 & 0,76 & 59,5 & 0,4 & 0,9996 \\
\hline 2,100 & $6,83 \times 10^{-3}$ a $2,54 \times 10^{-2}$ & 116,1 & 0,91 & 57,5 & 0,5 & 0,9993 \\
\hline 2,700 & $1,71 \times 10^{-2}$ a $2,54 \times 10^{-2}$ & 117,9 & 0,93 & 56,7 & 0,5 & 0,9992 \\
\hline 3,000 & $1,53 \times 10^{-2}$ a $2,54 \times 10^{-2}$ & 120,3 & 1,04 & 54,3 & 0,4 & 0,9989 \\
\hline
\end{tabular}

(a) Potencial formal da célula (valor médio de cinco repetições); (b) Desvio-padrão (valor médio, n=5); (c) coeficiente angular da curva analítica (valor médio, $\mathrm{n}=5$ ) ; (d) coeficiente de correlação (valor médio, $\mathrm{n}=5$ \} 
Com os dados das equações das curvas de calibração, chegou-se à seguinte relação matemática (equação 6):

$\mathrm{E}_{\mathrm{PAB}}{ }^{\circ}=104,9+5,0 \mathrm{I}$

onde:

$\left(\mathrm{E}^{\mathrm{O}^{\prime}}{ }_{\mathrm{PAB}}\right)_{\mathrm{o}}=(104,9 \pm 0,5) \mathrm{mv}$

A partir da equação (9) e do valor médio de $S=57,2 \pm 2,5 \mathrm{mV} /$ dec tem-se que:

$\mathrm{f}_{\mathrm{PAB}}=\log \mathrm{y}_{\mathrm{PAB}}=0,0874 \mathrm{I}$

Assim a equação (10) expressa a dependência matemática entre o coeficiente de atividade do íon p-aminobenzoato ( $\log \mathrm{y}_{\mathrm{PAB}}$ ) (na escala molar) e a força iônica (I) para o intervalo estudado.

O ajuste da equação (9) aos pontos $\left(\mathrm{E}_{\mathrm{PAB}}{ }^{{ }^{\circ} \text {, }}\right.$ I) para o sistema $\mathrm{NaPAB}-\mathrm{NaClO}_{4}$ pode ser verificado na figura 2.

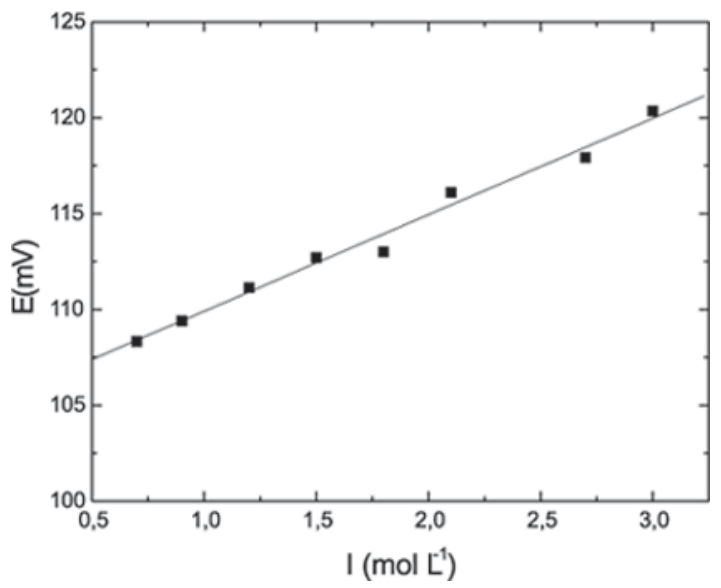

Figura 2 - Representação dos valores de $\mathrm{E}^{\circ}(\mathrm{mV})$ da tabela 1 em função da força iônica para o sistema $\mathrm{NaPAB}-\mathrm{NaClO}_{4}$ no intervalo de força iônica $\left(0,700\right.$ a 3,000) $\mathrm{mol} \mathrm{L}^{-1}$. Quadrados $=$ dados experimentais; curva contínua = equação ajustada: $\mathrm{E}$ ${ }^{\circ}{ }_{\mathrm{PAB}}=(104,9 \pm 0,5)+(5,00 \pm 0,26) \mathrm{I}, \quad(\mathrm{r}=0,9923)$.

Utilizando-se a equação (10) e calculando $\mathrm{y}_{\mathrm{PAB}}$ para cada valor de força iônica, obtiveram-se uma estimativa dos respectivos valores dos coeficientes de atividade iônica individuais, para o íon p-aminobenzoato em meio de $\mathrm{NaClO}_{4}$ aquoso, no intervalo de força iônica estudado, conforme mostram os dados da tabela 2.
Tabela 2 - Valores dos coeficientes de atividade $\mathrm{y}_{\mathrm{PAB}}$ no intervalo de força iônica $\mathrm{I}=(0,700-3,000) \mathrm{mol} \mathrm{L}^{-1}$ em meio de $\mathrm{NaClO}_{4}$ (aq.) e $\mathrm{T}=(25,0 \pm 0,1)^{\circ} \mathrm{C}$.

\begin{tabular}{cc}
\hline $\mathrm{I}\left(\mathrm{mol} \mathrm{L}^{-1}\right)$ & $\mathrm{y}_{\mathrm{pab}}{ }^{(\mathrm{a})}$ \\
\hline 0,7 & 1,2 \\
0,9 & 1,2 \\
1,2 & 1,3 \\
1,5 & 1,4 \\
1,8 & 1,4 \\
2,1 & 1,5 \\
2,7 & 1,7 \\
3,0 & 1,8 \\
\hline
\end{tabular}

(a) Valores calculados a partir da equação (10): $\log \mathrm{y}_{\mathrm{PAB}}=$ 0,0874 I

A literatura [18,36] preconiza que em casos de força iônica elevada os coeficientes de atividades para algumas espécies crescem e podem tornar-se maior que a unidade. Observando-se os dados da Tabela 2, verifica-se que os coeficientes de atividade são maiores que a unidade e que o eletrólito inerte $\left(\mathrm{NaClO}_{4}\right)$ exerce um efeito salino apreciável sobre o coeficiente de atividade do íon p-aminobenzoato. Comportamento semelhante foi observado para os sistemas $\mathrm{HAc}-\mathrm{NaAc}-\mathrm{NaClO}_{4}$ [27] $\mathrm{e} \mathrm{HClO}_{4}-\mathrm{NaClO}_{4}[4,32]$.

Não deve causar estranheza o fato de que o comportamento dos $\mathrm{y}_{\mathrm{PAB}}$ em soluções salinas concentradas de $\mathrm{NaClO}_{4}$ não seja o mesmo que o predito pela teoria de Debye-Hückel em soluções aquosas diluídas. No sistema estudado (NaPAB $\mathrm{NaClO}_{4}, \mathrm{I}=0,700$ - 3,000 mol L-1 $)$ o solvente não é exatamente a $\mathrm{H}_{2} \mathrm{O}$, mas uma mistura de $\mathrm{H}_{2} \mathrm{O}$ e $\mathrm{NaClO}_{4}$ e outros tipos de interações como as interações íon-solvente, bem como as especificidades dessas interações devem ser consideradas $[9,11,17,37]$. Portanto, o modelo de Debye - Hückel, seja na forma de sua equação clássica, seja na forma de suas equações estendidas, não é adequado para interpretar o comportamento de tais sistemas. Devido a dificuldade de se interpretar o comportamento de sistemas envolvendo misturas de eletrólitos em força iônica elevada, Gordon [14] propõe que o coeficiente de atividade de uma espécie i na presença de um excesso de um 
sal inerte $\left(\mathrm{y}_{\mathrm{i}}\right)$ é melhor avaliado por uma equação empírica do tipo da equação (5) empregada nesse trabalho. Estudos realizados por nosso grupo de pesquisa com sistemas similares ao estudado nesse trabalho demonstraram que a equação empírica proposta por Gordon é bastante adequada para se estimar os $y_{i}$ em tais sistemas [6,27,28,30-33].

É importante ressaltar que por um longo tempo a avaliação de propriedades individuais iônicas foi desencorajada, em virtude de prevalecer a opinião ortodoxa de que quantidades inacessíveis por termodinâmica exata como as atividades, energias de solvatação, etc. eram desprovidas de significado físico e utilidade prática [16]. A ampla utilização de uma quantidade "inexata" como o pH contribuiu mais que qualquer outro fator para a reavaliação dessa posição extrema [2] .

Os proponentes da "termodinâmica individual iônica" concordam que propriedades de íons individuais tais como atividades, coeficientes de atividades, energias de solvatação etc. não podem ser "exatamente avaliadas". Todavia, eles defendem uma estimativa das mesmas, alicerçados no fato de que tal estimativa pode fornecer valiosas informações físico-químicas que não podem ser obtidas por outros meios. Um dos grandes defensores do restabelecimento do significado físico de atividades individuais iônicas foi Frank [12]. Segundo o referido autor, apesar das incertezas inerentes envolvidas na estimativa de propriedades individuais iônicas as vantagens mostram-se consideráveis na reabertura da investigação e interpretação dos fenômenos de solução iônica.

$\mathrm{Na}$ opinião de Strehlow [38], a “termodinâmica individual iônica" é um ramo legítimo da físico-química já que provou ser capaz de fornecer predições e correlações corretas de fatos experimentais. Tal opinião foi referendada por trabalhos posteriores de renomados pesquisadores como Conway [8], Trasatti [15], Umezawa [40], Sammartano $[4,10]$,etc.

Os resultados obtidos por diversos autores $[4,10,29,40]$ e por nosso grupo de pesquisa [6,27,28,30-33] demonstram cabalmente que o coeficiente de atividade de um íon individual pode ser estimado por um eletrodo íon seletivo.

Por meio da equação (7), foi possível determinar o valor do potencial padrão do eletrodo indicador, $\mathrm{E}_{\mathrm{Hg} 2(\mathrm{PAB}) 2^{1 / 2 \mathrm{Hg}}}^{\circ}=(445,4 \pm 0,5) \mathrm{mV}$ a $(25,0$ $\pm 0,1)^{\circ} \mathrm{C}$, que convertido para escala molal, resultou em $\mathrm{E}^{\mathrm{o}}{ }_{\mathrm{Hg} 2(\mathrm{PAB}) 2 \frac{1}{2} \mathrm{Hg}}=(445,5 \pm 0,5) \mathrm{mV}$ a $(25,0 \pm$ $0,1)^{\circ} \mathrm{C}$. A partir desse valor, do valor de $\left.\mathrm{E}_{\mathrm{Hg}}^{\circ}{ }^{2+}\right|_{\mathrm{Hg}}$ $(790 \mathrm{mV}){ }^{28}$ foi possível através da equaçã̃o (8) determinar a constante termodinâmica do produto de solubilidade do p-aminobenzoato mercuroso, ${ }^{\mathrm{T}} \mathrm{K}_{\mathrm{ps}}$, (Tabela 3), que é inédita na literatura indexada.

Tabela 3 - Valores obtidos experimentais para o potencial padrão do eletrodo indicador e para a constante termodinâmica do produto de solubilidade do p-aminobenzoato mercuroso ( $\left.{ }^{\mathrm{T}} \mathrm{K}_{\mathrm{ps}}\right) \mathrm{à} \mathrm{tem-}$ peratura de $(25,0 \pm 0,1)^{\circ} \mathrm{C}$.

\begin{tabular}{ccc}
\hline $\mathbf{E}^{\mathrm{o}}{ }_{\mathrm{Hg} \text { (PAB)2 } \mid \mathrm{Hg}}$ & ${ }^{\mathrm{T}} \mathbf{K}_{\mathrm{ps}}$ & $\mathbf{p}^{\mathrm{T}} \mathbf{K}_{\mathrm{ps}}$ \\
\hline $445,4 \pm 0,5^{\mathrm{a}} ; 445,5 \pm 0,5^{\mathrm{b}, \mathrm{c}}$ & $2,50 \times 10^{-12} \mathrm{~mol}^{3} \mathrm{~L}^{-3}$ & 11,60 \\
\hline
\end{tabular}

a) Potencial padrão do eletrodo de p-aminobenzoato a $25^{\circ} \mathrm{C}$ vs eletrodo padrão de hidrogênio na escala molar considerando desprezível a contribuição do potencial de junção líquida.

b) Potencial padrão do eletrodo de p-aminobenzoato a $25^{\circ} \mathrm{C}$ vs eletrodo padrão de hidrogênio na escala molal considerando desprezível a contribuição do potencial de junção líquida.

c) Na literatura é comum expressar o valor do potencial padrão de um determinado eletrodo na escala molal. Segundo Covington e col. [7] para eletrodos de mercúrio - carboxilatos mercurosos mostra-se que em água a $25^{\circ} \mathrm{C}$, tem-se: $\mathrm{E}_{\mathrm{w}}^{\mathrm{o}}-\mathrm{E}_{\mathrm{m}}^{\mathrm{o}}=$ $0,15 \mathrm{mV}$, onde: subscrito $\underline{\mathrm{m}}$ refere-se à molaridade e subscrito $\underline{\text { w }}$ refere-se à molalidade.

Os resultados descritos na tabela 2 e na tabela 3 , demonstram claramente que o eletrodo de p-aminobenzoato, mostra-se bastante adequado para fornecer uma estimativa dos $\mathrm{y}_{\mathrm{PAB}}$ (coeficientes de atividade do íon p-aminobenzoato) na presença de um excesso de eletrólito inerte $\left(\mathrm{NaClO}_{4}\right)$ ,bem como, na determinação de parâmetros físicoquímicos como o ${ }^{\mathrm{T}} \mathrm{K}_{\mathrm{ps}}$ do p-aminobenzoato mercuroso e o $\mathrm{E}^{\mathrm{o}}{ }_{\mathrm{Hg} 2(\mathrm{PAB}) 2^{1 / 2 \mathrm{Hg}}}$ (potencial padrão do eletrodo na escala molal vs eletrodo padrão de hidrogênio).

Um outro aspecto importante é que o eletrodo desenvolvido pode ser aplicado em estudos quantitativos de equilíbrios em solução, uma vez que ele pode ser utilizado para se determinar a concentração de ligante livre ( $[\mathrm{L}]=[\mathrm{p}-$ aminobenzoato] ) em meio iônico constante (isto é, quando I = constante e o eletrólito de suporte 
está presente em concentração suficientemente majoritária). Conhecendo-se o valor de [L] é possível o acesso direto às constantes de estabilidade de complexos envolvendo p-aminobenzoato e íons metálicos.

Além disso,o emprego dos $\mathrm{y}_{\mathrm{PAB}}$ juntamente com parâmetros anteriormente obtidos ${ }^{30,32}\left(\mathrm{y}_{\mathrm{H}}, \mathrm{y}_{\mathrm{Pb}}\right.$, $\mathrm{y}_{\mathrm{Cd}}, \mathrm{y}_{\mathrm{Cu}}$ ), abre várias possibilidades em estudos de equilíbrio de complexos em solução,tais como:

a) Encontrar equações matemáticas simples que possibilitem a interconversão das constantes de estabilidade de complexos binários 1:1 envolvendo p-aminobenzoato e alguns metais bivalentes para qualquer valor de I dentro do intervalo estudado $\left(0,700\right.$ a 3,00) $\mathrm{mol} \mathrm{L}^{-1} \cdot[30-32]$

b) Estimar as constantes termodinâmicas dos referidos complexos .[30,32]
Outro aspecto relevante é o fato do referido eletrodo monitorar diretamente as atividades do íon p-aminobenzoato, o que possibilita que medidas farmacocinéticas (metabolismo, excreção urinária, etc...) e de biodisponibilidade sejam realizadas.

Estudos visando o emprego analítico desse eletrodo na determinação de PABA em formulações farmacêuticas, encontram-se atualmente em andamento.

\section{Agradecimentos}

Os autores agradecem a Fapesp e a Fundunesp pelos suportes financeiros ao grupo de pesquisa e ao CNPq pelas bolsas concedidas.

Recebido em: 29/05/03 Aceito em: 17/07/03

A. O. Santini; E. S. da Silva Júnior; H. R. Pezza; L. Pezza. Determination of physical chemical parameters with a potentiometric sensor sensitive to p-aminobenzoate ion.

Abstract: The paper describes the construction and determination of physical chemical parameters with a second kind electrode, $\mathrm{Pt}|\mathrm{Hg}| \mathrm{Hg}_{2}$ (PABA) $\mid$ Grafite, sensitive to p-aminobenzoate ion (PAB). Electrode construction is easy, presents a fast response time $(30 \mathrm{sec})$, low-cost, excellent response stability and (lifetime > 12 months, under continuous use).

Using of the mercury(I) p-aminobenzoate electrode, the molar single activity coefficient associated with $\mathrm{PAB}\left(\mathrm{y}_{\mathrm{PAB}}\right)$ was estimated at $25^{\circ} \mathrm{C}$ and ionic strengths between $0.700-3.000 \mathrm{~mol} \mathrm{~L}^{-1}\left(\mathrm{NaClO}_{4}\right)$. The standard molal potential of the electrode at $25^{\circ} \mathrm{C}$ is $445.5 \pm 0.5 \mathrm{mV}$.

The thermodynamic solubility-product constant of mercurous p-aminobenzoate determined with the mentionated electrode, in aqueous solution, at $25^{\circ} \mathrm{C}$ was ${ }^{\mathrm{T}} \mathrm{K}_{\mathrm{ps}}=2.50 \times 10^{-12} \mathrm{~mol}^{3} \mathrm{~L}^{-3}$.

An important feature of the electrode is that it permits the determination of the concentration of free $\mathrm{p}$ aminobenzoate ions in complex systems of metallic ions.

Keywords: potentiometric sensor; p-aminobenzoate; thermodynamic solubility-product constant; activity coefficients.

\section{Referências}

[1] A. J. Antunes, V. P. Canhos, Aditivos em Alimentos, Campinas, Secretaria da Indústria, Comércio, Ciência e Tecnologia - Coordenadoria e Comércio, 1989, p. 125.

[2] R. G. Bates, Medium effects and $\mathrm{pH}$ in non-aqueous solvents, In: J. F. Coetzee, C. D. Ritchie, Eds, Solute-Solvent Interactions, Marcel Dekker, New York, 1969, chap. 2.

[3] M. Bruze, B. Gruvberger, I. Thulin, Photodermatol. Photoimmunol. Photomed. 7 (1990) 106.
[4] S. Caponi, A. de Robertis, C. de Stefano, S. Sammartano, R. Scarcella, Talanta 34 (6) (1987) 593.

[5] T. Carpenter, A. O'Connor, J. Orfanelli, R. Siegfried, Drug Cosmet. Ind. 158 (3) (1996) 56.

[6] A. C. V. Cavalheiro, M. de Moraes, L. Pezza, Eclét. Quím. 25 (2000) 123.

[7] A. K. Convington, P. K. Talukdar, H. R. Thirsk, Trans Faraday Soc. 60 (1964) 412.

[8] B. E. Conway, J. Sol. Chem. 7 (10) (1978) 721. 
[9] B. E. Conway, Ionic interactions and activity behavior of electrolyte solutions, In: B. E. Conway, J. O'm Bockris, E. Yeager, Eds, Comprehensive Treatise of Electrochemistry, Plenum Press, New York, 1983, v. 5, p. 111.

[10] P. G. Daniele, C. De Stefano, C. Foti, S. Sammartano, Curr. Top. Solution Chem. 2 (1997) 253

[11] H. S. Frank, Z. Phys. Chem. 228 (1965) 364.

[12] H. S. Frank, J. Phys. Chem. 67 (1963) 1554.

[13] L. S. Goodman, A. Gilman, The Pharmacological Basis of Therapeutics, Mc Graw-Hill , 2001, 10th edn., Section XIII, p. 2148.

[14] J. E. Gordon, The Organic Chemistry of Electrolyte Solutions, Wiley, New York, 1975, p. 36.

[15] R. Grassi, A. Daghetti, S. Trasatti, J. Electroanal. Chem. 226 (1987) 341.

[16] E. A. Guggenheim, J. Phys. Chem. 33 (1929) 842.

[17] R. W. Gurney, Ionic Process in Solution, Mcgraw-Hill, New York, 1953, p. 163.

[18] D. C. Harris, Quantitative Chemical Analysis, W. H. Freeman, New York, 1999, p. 178.

[19] II. Antimicrobial Drugs. A. Synthetic chemotherapeutic agents. 1. Sulfonamides. Disponível em: http:// www.mbc.pharm.utoledo.edu/mbc/ undergradcourses/ 3800notes/3800_j10.htm>. Acesso em: ago. 2002.

[20] K. Ikeda, S. Suzuki, Y. Watanabe, J. Chromatogr. 482 (1989) 240

[21] K. Ikeda, S. Suzuki, Y. Watanabe, J. Chromatogr. 513 (1990) 321.

[22] W. D. Larson, F. H. Macdougall, J. Phys. Chem. 41 (1937) 493.

[23] J. J. Lingane, Anal. Chem. 40 (1968) 935.

[24] M. O. Masse, H. Borremans, Int. J. Cosmet. Sci. 13 (1991) 303

[25] M. Molina, C. B. Melios, Quím. Nova 11(3) (1988) 291. [26] D. C. Moore, G. Davies, Anal. Chem. 47 (1975) 2477.
[27] M. de Moraes, M. Molina, C. B. Melios, J. O. Tognolli, L. Pezza, Quím. Nova 17(1) (1994) 35.

[28] M. de Moraes, L. Pezza, C. B. Melios, M. Molina, H. R. Pezza, A. M. Peres, A. C. Villafranca, J. Carloni, Eclét. Quím. 21 (1996) 133.

[29] El-Ichiro Ochiai, J. Chem. Educ. 67 (6) (1990) 489.

[30] L. Pezza, M. Molina, C. B. Melios, M. de Moraes, J. O. Tognolli, H. M. Gomes, Int. J. Environ. Anal. Chem. 68 (3) (1997) 295

[31] L. Pezza, M. Molina, C. B. Melios, M. de Moraes, J. O. Tognolli, Talanta 43 (1996) 1697.

[32] L. Pezza, M. Molina, M. de Moraes, C. B. Melios, J. O. Tognolli, Talanta 43 (1996) 1689.

[33] L. Pezza, Sistemas Binários Envolvendo Alguns Ions de Metais Bivalentes e Monocarboxilatos e Solução Aquosa. Estudo Sobre Relações Entre Constantes De Formação E A Força Iônica Do Meio. 236 p. Tese (Doutorado em Química) Instituto de Química, Universidade Estadual Paulista Júlio de Mesquita Filho, Araraquara, 1993.

[34] L. Pezza, A. O. Santini, H. R. Pezza, C. B. Melios, V. J. F. Ferreira, A. L. M. Nasser, Anal. Chim. Acta 433 (2) (2001) 281.

[35] E. P. Serjent, Potentiometry and Potentiometric Titrations, Wiley, New York, 1984, p. 618.

[36] A. D. Skoog, J. F. Holler, T. A. Nieman, Princípios de Análise Instrumental, Bookman, São Paulo, 2002, p. 769.

[37] J. Steigman, J. Dobrow, J. Phys. Chem. 72 (10) (1968) 3424 .

[38] H. Strehlow, Electrode Potentials In Non-Aqueous Solvents, In: J. J. Lagowski, The Chemistry of Non-Aqueous Solvents, Academic Press, New York, 1966, chap.4.

[39] F. P. Tomasella, P. Zutting, L. Cline, J. Chromatogr. 587 (1991) 325

[40] I. Uemasu, Y. Umezawa, Anal. Chem. 55 (1983) 386

[41] S. P. Wang, J. C. Won, Anal. Chim. Acta 416 (2000) 157. 